\title{
Prevalence and main indicators of the combination of resistant tuberculosis and diabetes mellitus
}

Todoriko L.D., Semianiv I.O.

Bukovinian State Medical University, Chernivtsi, Ukraine

Background. Diabetes is the background for the development of tuberculosis (TB) and such comorbidity not only significantly complicates the specific process, but also is one of the main risk factors for TB recurrence.

Objective. The aim of the study is a comprehensive retrospective assessment of the combination of TB and diabetes.

Materials and methods. Our study is based on an analysis of statistical data obtained from a retrospective study of 762 case histories.

Results. Depending on the type of TB case in our patients, we found that in both groups of the study the recurrence of TB prevailed - 49 cases (55.7\%) against 39 cases (44.3\%) of people in the main group; 363 cases (53.9\%) against $311(46.1 \%)$ in the control group $(p<0.05)$. The rate of successful treatment in group 2 is probably higher than in group 1 (64.7\% vs. $61.4 \%$ ). However, a more significant probable difference is characterized by the treatment rate, which in the main group is $27.3 \%$ vs $40.3 \%$ in the control group (almost 2 times). The rate of ineffective treatment, which in patients with comorbidity was $27.3 \%$ (almost every third patient) against $17.6 \%$ in group 2 is also important for scientists and practitioners.

Conclusions. The pulmonary TB developed significantly more often in middle-aged patients with type 2 diabetes mellitus with moderate and severe states, the subcompensated form, with a complicated course. In patients with diabetes more often was registered a common tuberculous process in the lungs and in all $100 \%$ of patients with syntropy bacterial excretion was registered. 